\title{
Les risques liés aux expositions au tritium sont-ils sous-évalués?
}

\author{
F. PAQUET ${ }^{1 *}$, H. MÉTIVIER ${ }^{2 *}$
}

(Manuscrit reçu le 26 mars 2008, accepté le 28 avril 2008)

RÉSUMÉ Le tritium est un radionucléide dont l'utilisation et la production vont croitre du fait du développement des nouveaux réacteurs à fusion et de la relance des programmes nucléaires dans le monde. La toxicité du tritium est bien connue mais ses effets sanitaires restent délicats à appréhender du fait d'une dosimétrie difficile et du faible nombre de cas de contamination survenus depuis son utilisation. L'estimation des risques liés à son utilisation repose sur les modèles de la CIPR qui permettent de calculer les doses délivrées dans les tissus grâce à un facteur de pondération $W_{R}$ qui tient compte de l'efficacité biologique relative des différents rayonnements. Quelques voix s'élèvent pour réclamer une révision du facteur de pondération utilisé pour les rayonnements bêta du tritium, arguant que celui-ci peut s'incorporer dans l'ADN et donc être plus toxique que les autres radionucléides émetteurs bêta. Une revue des différents travaux menés sur le sujet et publiés dans la littérature montre en réalité que l'efficacité biologique relative du tritium n'est pas très différente de celle des rayonnements gamma, pris comme rayonnements de référence. Ceci plaide pour un maintien du facteur de pondération unitaire actuel, initialement proposé puis réaffirmé récemment par la CIPR.

ABSTRACT Are the risks from tritium exposures underestimated?

Tritium is a radionuclide that will be further used and produced in fusion reactors. The tritium toxicity is well-known but its health consequences are more difficult to evaluate, due to difficulties for assessing doses and to the very few cases of contamination over the years. The assessment of risks resulting from tritium exposure is based on ICRP models that enable calculation of doses in the tissues, by the use of a weighting factor $W_{R}$, based on the relative biological effectiveness of the various radioactive emissions. Some authors are currently asking for a revision of the weighting factor to be used for the beta emission of tritium, based on its potential incorporation into DNA. A review of the recent relevant publications published on this subject shows that the relative biological effectiveness of tritium is not so different from that of gamma emissions, which are taken as reference radiations. This is in favour of keeping the current weighting factor of 1 for beta emissions of tritium, value initially proposed and then reaffirmed by the ICRP.

Keywords: tritium / EBR / $\mathbf{W}_{\mathbf{R}}$

Le tritium est un isotope radioactif de l'hydrogène. Il est produit naturellement par action des rayonnements cosmiques sur l'atmosphère (Grosse et al., 1951, 1954)

${ }^{1}$ IRSN, Direction scientifique, BP 3, 13115 Saint-Paul-lez-Durance Cedex, France.

${ }^{2}$ SFRP, BP 72, 92263 Fontenay-aux-Roses Cedex, France.

* respectivement membre et ancien membre du comité 2 de la CIPR 
mais aussi lors des essais d'armes nucléaires, et, dans une moindre mesure, dans les réacteurs nucléaires (UNSCEAR, 1977, 1982, 1993).

Sa toxicité potentielle, tant sur l'homme que sur l'environnement, a toujours fait l'objet d'une attention particulière eu égard à sa mobilité, son incorporation rapide dans les organismes et à son mode de décroissance radioactive, par émission bêta. Son action délétère sur les cellules et organismes vivants est bien connue et documentée (Hofer et Hughes, 1971 ; Pomerantseva et al., 1984 ; Balonov et al., 1993 ; Belot et al., 1996). On sait que le tritium peut être intégré dans des molécules organiques dont l'ADN et peut être carcinogène chez les animaux de laboratoire.

Ses effets chez l'homme sont plus difficiles à appréhender. De nombreuses études épidémiologiques ont été menées à la fois sur les travailleurs exposés, leur descendance et les membres du public. Ces études sont malheureusement difficilement exploitables en raison du manque de précision sur les doses reçues, voire même quelquefois sur la nature des effets observés (Beral et al., 1988 ; Green et al., 1997 ; Zablotska et al., 2004 ; Richardson et Wing, 2007 ; Little et Wakeford, 2008).

Malgré ces difficultés, quelques voix s'élèvent au sein de diverses associations pour affirmer que les risques sanitaires liés aux expositions au tritium sont sousévalués. Ces remarques viennent en complément d'un rapport récent établi par un groupe de scientifiques anglais qui réclament une révision des facteurs de pondération $\mathrm{W}_{\mathrm{R}}$ utilisés pour le calcul des doses délivrées par le tritium après incorporation (AGIR, 2007). De façon similaire, un autre groupe de scientifiques conclut que les doses reçues après incorporation de radionucléides, calculées selon les modèles de la CIPR, pourraient être sous estimées d'un facteur 10 (CERRIE, 2004).

Cette agitation récente pourrait donner le sentiment que la toxicité du tritium et plus particulièrement ses effets sanitaires sont méconnus. En réalité plusieurs centaines de publications sont parues sur le sujet ces toutes dernières années et font du tritium un des radionucléides les mieux étudiés. La remise en cause de sa toxicité répond manifestement aux inquiétudes liées à la relance des programmes nucléaires et du réacteur ITER, qui requiert une charge initiale de tritium pour fonctionner et qui en produira lui-même (Cristescu et al., 2007). Cette nouvelle source de tritium, qui restera confinée, génère des appréhensions légitimes et de nombreuses questions quant à sa toxicité et à son impact sur l'environnement.

Pour répondre à ces inquiétudes, plusieurs articles de synthèse viennent d'être publiés pour faire le point sur le comportement et la toxicité du tritium. Dans ce contexte, cet article vise à rappeler les connaissances acquises sur les effets 
biologiques et sanitaires du tritium et à rappeler comment sont évalués les risques et les coefficients de pondérations utilisés pour le calcul de dose. Il permettra de rappeler l'étendue des travaux menés et la position de la CIPR, qui s'est récemment prononcée sur le cas spécifique du tritium.

\section{L'estimation du risque après incorporation de tritium}

La méthode de choix pour évaluer le risque consécutif à l'incorporation de radionucléides est de mener des études épidémiologiques spécifiques et de relier le risque d'apparition de pathologies aux doses délivrées dans l'organisme ou, à défaut, aux quantités incorporées.

Lorsque les études épidémiologiques font défaut, une méthode alternative pour déterminer les risques sanitaires consécutifs à l'incorporation d'un radionucléide consiste à utiliser des modèles biocinétiques et dosimétriques qui permettront de calculer les doses délivrées après incorporation, puis de rapprocher les doses calculées des effets sanitaires observés chez les survivants de Hiroshima et Nagasaki. Dans le cas du tritium cette approche implique de définir la toxicité relative des émissions bêta du radionucléide par rapport aux rayonnements gamma émis à Hiroshima. Cette toxicité spécifique s'exprime in fine à l'aide d'un coefficient de pondération $\mathrm{W}_{\mathrm{R}}$, utilisé pour calculer la dose aux organes. Cette approche, bien que communément utilisée dans de nombreuses situations est quelquefois critiquée (EGIS, 2007) et particulièrement dans le cas du tritium, du fait de sa diffusion au sein même des molécules d'ADN et donc de sa forte toxicité potentielle.

La difficulté, pour traiter la question des risques sanitaires associés à l'incorporation de tritium, vient du fait que, en radioprotection, l'évaluation des risques repose sur la probabilité d'apparition d'effets stochastiques. De fait, les facteurs de pondération $\mathrm{W}_{\mathrm{R}}$ définis par la CIPR sont utilisés pour une analyse prospective du risque, basée sur l'apparition d'effets stochastiques, donc essentiellement des effets héréditaires et des cancers. Ces facteurs de pondération des rayonnements ont donc été choisis pour être représentatifs des valeurs de l'efficacité biologique relative (EBR) des rayonnements considérés pour le déclenchement d'effets stochastiques à doses faibles (ICRP, 1991). Dans le cas du tritium, émetteur d'électrons, le facteur de pondération préconisé est le même que celui utilisable pour toutes les autres sources émettrices d'électrons, de muons et de photons et est égal à une valeur unitaire (ICRP, 1991, 2007). La CIPR reconnaît que la toxicité des électrons du tritium incorporé dans des précurseurs de l'ADN peut être différente, mais, en l'absence de données définies dans le domaine, vise à offrir un modèle simple de gestion pour l'appréciation prospective des risques et recommande donc l'utilisation d'un facteur unique de pondération (ICRP, 1991). 
Néanmoins, dans le cas d'estimation individuelle et rétrospective du risque, elle recommande l'utilisation de données physiologiques et biophysiques spécifiques lorsque celles-ci sont disponibles (ICRP, 2007).

Remettre en question le facteur de pondération du tritium nécessite donc de revoir son EBR mais en se limitant aux valeurs obtenues pour l'apparition de cancers, ou, à défaut à ceux déterminés pour l'apparition de phénomènes initiaux pouvant conduire à l'apparition d'effets stochastiques. La revue des études menées montre que, malgré ces conditions très restrictives, de nombreuses études sont exploitables.

\section{L'efficacité biologique relative du tritium}

Les valeurs d'efficacité biologique relative du tritium reportées dans les différentes publications scientifiques varient selon les auteurs. En fait, l'EBR d'un radionucléide dépend de nombreux paramètres qui doivent être soigneusement répertoriés avant toute utilisation des résultats.

En tout premier lieu, les EBR, définies par rapport à un rayonnement de référence dépendent des caractéristiques de ce rayonnement. Les rayonnements de référence sont en général des photons de faible transfert linéique d'énergie mais, dans la publication 60 de la CIPR (ICRP, 1991), aucune énergie de référence n'a été définie et dans les faits les rayonnements de référence utilisés ont été soit des émissions gamma produites par du césium- 137 ou du cobalt-60, soit des rayons $\mathrm{X}$ de $200 \mathrm{kV}$ (ICRP, 1991). Ce point semble anodin mais influe fortement sur la valeur de l'EBR déterminé de façon expérimentale. Il est connu que les rayons $\mathrm{X}$ de $250 \mathrm{kV}$ sont deux fois plus efficaces, par unité de dose, pour produire des effets biologiques, que les rayons gamma du cobalt-60 (Straume, 1995). Avant toute discussion sur les EBR du tritium il conviendra donc de distinguer ceux obtenus par référence aux rayons $X$ de ceux obtenus par référence aux rayonnements gamma du cobalt ou du césium.

L'EBR des éléments varie avec la dose, le débit de dose utilisé et, plus généralement, avec la durée d'exposition (Rossi and Zaider, 1990 ; Ujeno, 1983 ; Edwards, 1999). Il convient donc, lorsque cela est possible, de définir un $\mathrm{EBR}_{\max }$ en fonction de la dose (ICRU, 1986) qui devra être utilisé. Ces données ne sont pas toujours disponibles car elles sont souvent obtenues pour une dose unique et cela rend difficile l'interprétation des données publiées. De plus, dans de nombreuses études, les EBR du tritium incorporé dans des organismes et donc délivrant des doses de façon chronique, ont été obtenus par comparaison avec des rayonnements $\mathrm{X}$ ou gamma externes émis de façon aigue. Ces données sont donc difficilement utilisables et peuvent largement fausser la validité des résultats. 
Les EBR dépendent des systèmes biologiques étudiés, des conditions d'expérimentations et, surtout, des effets recherchés. Il n'est donc pas possible d'agréger l'ensemble des EBR déterminés pour le tritium et, pour le cas spécifique de la radioprotection et de la détermination des facteurs de pondération des rayonnements, il conviendra de se limiter aux EBR obtenus pour l'apparition de cancers, ou, à défaut à ceux déterminés pour l'apparition de phénomènes initiaux pouvant conduire à l'apparition d'effets stochastiques. Ces derniers effets peuvent être, sous certaines conditions, les mutations cellulaires, les aberrations chromosomiques et l'acquisition d'un état d'instabilité génétique. L'utilisation de ces données doit toutefois être menée avec de nombreuses réserves car ces effets ne représentent qu'une des étapes pouvant conduire à la cancérogénèse et ne tiennent pas compte de l'ensemble des phénomènes de la cancérogénèse et notamment des phénomènes de régulation et d'homéostasie tissulaire (BarcellosHoff et Brooks, 2001).

Enfin, dernier point, l'efficacité biologique relative du tritium va dépendre de sa localisation dans les cellules. Il est admis que le tritium incorporé dans des précurseurs de l'ADN (ex thymidine, deoxycytidine, ...) et dans les précurseurs des histones (arginine) peut délivrer des doses à l'ADN plus importantes que lorsque qu'il est localisé en dehors du noyau cellulaire comme c'est le cas après incorporation d'eau tritiée (Streffer et al., 1978). La logique serait donc de définir des EBR puis des facteurs de pondération pour ces différents cas de figure.

Malgré ces restrictions, de nombreux auteurs ont tenté de déterminer les EBR du tritium. Environ 200 études ont été réalisées ces 30 dernières années, visant à déterminer l'EBR de ce radionucléide dans de très nombreuses configurations et pour l'apparition de très nombreux effets différents dont les aberrations chromosomiques, la formation de micronoyaux, les transformations cellulaires, la survie cellulaire des spermatocytes et ovocytes et les effets tératogènes. Ces études ont été analysées et résumées à différentes occasions (Straume et Carsten, 1993 ; ICRP, 2007 ; Little et Lambert, 2008). Pour utilisation en radioprotection, les seules études qui pourront être retenues seront celles portant sur l'apparition de tumeurs et réalisées par rapport à une exposition chronique à faible dose de rayonnement gamma ou $\mathrm{X}$.

Lorsque le tritium est utilisé sous forme d'eau tritiée, les EBR déterminées dans des études de cancérogénèse sont généralement comprises entre 1 et 5 lorsque le rayonnement de référence est un rayonnement gamma délivré de façon chronique et entre 1,1 et 2,5 lorsque le rayonnement de référence est un rayonnement X délivré de façon chronique (Seyama et al., 1991 ; Zhou et al., 1989 ; Satow et al., 1989 ; Matsuda et al., 1986 ; Revina et al., 1984 ; Gragtmans et al., 1984 ; Johnson et al., 1995). 
Cependant, cet intervalle de distribution ne peut être utilisé en l'état car il rassemble les valeurs extrêmes sans tenir compte de la fréquence des différentes valeurs. Pour pallier cet état de fait, Little et Lambert (2008) ont analysé l'ensemble des études citées et ont tenté de calculer une «meilleure estimation » des $\mathrm{EBR}_{\max }$ à partir d'une sélection de données. Les auteurs ont ainsi sélectionné les études publiées dans des revues à comité de lecture, éliminé les publications pour lesquelles les analyses statistiques paraissaient inadéquates et calculé pour différents effets, différents rayonnements de références et différents modes expérimentaux, une EBR basée sur l'agrégation des études sélectionnées. Ces auteurs ont ainsi montré que l'EBR pour l'apparition de tumeurs était centrée autour de 2,5 par rapport aux rayons gamma et de 1,2 par rapport aux rayons $\mathrm{X}$ délivrés de façon chronique, soit dans des conditions relativement similaires à celles prévalant pour le tritium (Little et Lambert, 2008).

Lorsque l'ensemble des études menées in vivo sont agrégées (quel que soit donc l'effet recherché), les EBR sont centrés sur 1,6 par rapport aux rayonnements gamma et sur 1,2 pour les rayonnements $\mathrm{X}$, délivrés de façon chronique.

Ces études sont très informatives mais doivent être maniées avec précaution. La méthode utilisée par ces auteurs pour agréger un EBR standard peut être critiquée, notamment au niveau de la méthode de calcul et de la sélection des études utilisées dans le calcul. De fait, la CIPR préfère dans sa dernière publication se baser sur l'ensemble des valeurs publiées et estimer la gamme d'EBR prédominantes. Elle conclut ainsi que la plupart des EBR, tous effets confondus sont compris entre 1 et 3 par rapport aux rayons gamma et entre 1 et 1,5 pour les rayons $\mathrm{X}$ (ICRP, 2007).

\section{De l'EBR au facteur de pondération des rayonnements}

L'utilisation et l'exploitation de l'ensemble de ces EBR pour dériver un facteur de pondération pour les rayonnements beta du tritium sont éminemment délicates.

En toute logique, il conviendrait, toujours dans le cadre de la radioprotection et de celui de l'utilisation des coefficients de risque tirés du suivi des survivants de Hiroshima et Nagasaki, d'utiliser préférentiellement les EBR obtenues par rapport à un rayonnement gamma puisque ce type de rayonnement a été celui délivré lors des explosions des bombes atomiques. Pour ce faire, il faudra néanmoins se souvenir que les énergies des rayons gamma des sources usuellement utilisées (cobalt-60 et césium-137) dans les études des EBR sont plus faibles que les énergies délivrées par les rayons gamma de Hiroshima et Nagasaki (HN) (0,6 MeV pour ${ }^{137} \mathrm{Cs}$ et 1,17 et $1,33 \mathrm{MeV}$ pour ${ }^{60} \mathrm{Co}$ contre $2-5 \mathrm{MeV}$ en moyenne pour $\mathrm{HN}$ (Young et Kerr, 1995)) et qu'aucune de ces études ne pourra être utilisée sans inclure un certain nombre d'incertitudes. 
De fait, la CIPR préfère conclure que les valeurs des EBR trouvées pour le tritium incorporé sous forme d'eau tritiée ne diffèrent pas notablement de celles trouvées pour les autres radiations à faible transfert linéique d'énergie et recommande, par conséquent, d'utiliser un facteur de pondération $\mathrm{W}_{\mathrm{R}}$ unique de 1 , afin de simplifier les calculs qui sont dédiés, doit on le rappeler, à une estimation prospective du risque (ICRP, 2007).

Dans le cas du tritium incorporé sous forme organique les choses peuvent être plus complexes et sont nettement moins documentées. Les quelques valeurs obtenues expérimentalement semblent montrer que les EBR obtenues après incorporation de tritium sous forme d'acides aminés marqués sont similaires à celles obtenues après incorporation d'eau tritiée (Ueno et al., 1989). Par contre, lorsque le tritium est incorporé sous forme de thymidine tritiée, incorporable à l'ADN, les quelques résultats acquis sont contradictoires. L'EBR pour la mortalité cellulaire et la mutation in vitro de cellules de souris est deux fois plus importante que celle obtenue avec l'eau tritiée (Ueno et al., 1989) mais moins importante lorsqu'il s'agit de la survie de spermatogonies (Lambert, 1969). D’autres expériences menées in vitro sur des embryons de souris avant implantation montrent, quant à elles, que la thymidine tritiée peut être jusqu'à 10000 fois plus toxique que l'eau tritiée (Müller et al, 1986). L'ensemble de ces études montre que la détermination d'une EBR est complexe et pose la question de l'extrapolation d'un effet biologique observé sur une cellule à un effet stochastique dont on veut se protéger.

\section{Conclusions}

Les études traitant de la toxicité et des effets sanitaires du tritium sont menées et réévaluées en continu. Le calcul des EBR du tritium fait apparaitre des valeurs variables selon les effets recherchés. Lorsque ceux-ci se limitent aux effets stochastiques, pour lesquels le système de radioprotection a émis des outils de gestion, les valeurs de ces EBR sont proches de 1. De fait, la CIPR a confirmé dans ses dernières recommandations que le facteur de pondération $\mathrm{W}_{\mathrm{R}}$ du tritium devait être de 1, similaire donc à celui des rayonnements électromagnétiques (ICRP, 2007). La CIPR recommande néanmoins que, dans le cas d'études dosimétriques rétrospectives individuelles, une analyse spécifique de risque soit réalisée, avec utilisation de données biophysiques et physiologiques individuelles. De même, la CIPR reste attentive à l'évolution des travaux menés dans le domaine. Dans ce cadre, le comité 2 de la CIPR a examiné lors de sa dernière réunion les travaux du groupe AGIR publiés en 2007 mais a considéré que ce rapport ne présentait pas de nouvelles données nécessitant de revisiter cette conclusion. 


\section{RÉFÉRENCES}

AGIR (2007) Review of Risks from Tritium, Report of the independent Advisory Group on Ionising Radiation, Documents of the Health Protection Agency, Radiation, Chemical and Environmental Hazards, REC-4. November 2007. ISBN: 978-0-85951-610-5.

Balonov M.I., Muksinova K.N., Mushkacheva G.S. (1993) Tritium radiobiological effects in mammals: review of experiments of the last decade in Russia, Health Phys. 65, 713-726.

Barcellos-Hoff M.H., Brooks A.L. (2001) Extracellular signaling through the microenvironment: a hypothesis relating carcinogenesis, bystander effects, and genomic instability, Radiat. Res. 156, 618-627.

Belot Y., Roy M., Métivier H. (1996) Le tritium, de l'environnement à l'homme, Collection IPSN, EDP Sciences, les Ulis. ISBN: 2-86883-275-X.

Beral V., Fraser P., Carpenter L., Booth M., Brown A., Rose G. (1988) Mortality of employees of the Atomic Weapons Establishment, 1951-82, Br. Med. J. 297, 757-770.

CERRIE (2004) Report of the Committee Examining Radiation Risks of Internal Emitters, Produced by CERRIE, London. ISBN: 0-85951-545-1.

Cristescu I., Cristescu I., Doerr L., Glugla M., Murdoch D. (2007) Tritium inventories and tritium safety design principles for the fuel cycle of ITER, Nuclear Fusion 47, S458-S463.

Edwards A.A. (1999) Neutron RBE values and their relationship to judgements in radiological protection, J. Radiol. Prot. 19, 93-105.

EGIS (2007) Scientific Issues and Emerging Challenges for Radiological Protection, In NEA report $\mathrm{N}^{\circ} 6167$ (NEA OECD Ed.) pp. 1-119.

Gragtmans N.J., Myers D.K., Johnson J.R., Jones A.R., Johnson L.D. (1984) Occurrence of mammary tumors in rats after exposure to tritium beta rays and 200-kVp X rays, Radiat. Res. 99, 636-650.

Green L.M., Dodds L., Miller A.B., Tomkins D.J., Li J., Escobar M. (1997) Risk of congenital anomalies in children of parents occupationally exposed to low level ionising radiation, Occup. Environ. Med. 54, 629-635.

Grosse A.V., Johnston W.M., Wolfgang R.L., Libby W.F. (1951) Tritium in Nature, Science 113, 1-2.

Grosse A.V., Kirschenbaum A.D., Kulp J.L., Broecker W.S. (1954) The natural tritium content of atmospheric hydrogen, Phys. Rev. 93, 250-251.

Hofer K.G., Hughes W.L. (1971) Radiotoxicity of intranuclear tritium, 125 iodine and 131 iodine, Radiat. Res. 47, 94-101.

ICRP Publication 60 (1991) 1990 Recommendations of the International Commission on Radiological Protection, Ann. ICRP 21(1-3).

ICRP Publication 103 (2007) The 2007 recommendations of the International Commission on Radiological Protection, Ann. ICRP 37(2-4).

ICRU (1986) The quality factor in radiation protection. ICRU report 40 Bethesda.

Johnson J.R., Myers D.K., Jackson J.S., Dunford D.W., Gragtmans N.J., Wyatt H.M., Jones A.R., Percy D.H. (1995) Relative biological effectiveness of tritium for induction of myeloid leukemia in CBA/H mice, Radiat. Res. 144, 82-89.

Lambert B.E. (1969) Cytological damage produced in the mouse testes by tritiated thymidine, tritiated water and X-rays, Health Phys. 17, 547-557.

Little M.P., Lambert B.E. (2008) Systematic review of experimental studies on the relative biological effectiveness of tritium, Radiat. Environ. Biophys. 47, 71-93.

Little M.P., Wakeford R. (2008) Systematic review of epidemiological studies of exposure to tritium, J. Radiol. Prot. 28, 9-32.

Matsuda Y., Yamada T., Tobari I. (1986) Chromosome aberrations induced by tritiated water or 60Co gamma-rays at early pronuclear stage in mouse eggs, Mutat. Res. 160, 87-93. 
LES RISQUES LIÉS AUX EXPOSITIONS AU TRITIUM SONT-ILS SOUS-ÉVALUÉS ?

Müller W.U., Streffer C., Molls M., Glück L. (1986) Radiotoxicity of ${ }^{3} \mathrm{H}$-Thymidine and ${ }^{3} \mathrm{H}$-arginine in pre-implantation mouse embryos in vitro, Radiat. Prot. Dos. 16, 155-158.

Pomerantseva M.D., Balonov M.I., Ramaiia L.K., Vilkina G.A. (1984) Mutagenic action of tritium on the germ cells of male mice. II. The genetic damages in stem spermatogonia induced by tritium oxide and gamma radiation, Genetika 20, 782-787.

Revina V.S., Voronin V.S., Lemberg V.K., Sukhodoev V.V. (1984) Comparative evaluation of the carcinogenic effects of chronic exposure to tritium oxide and external gamma-radiation, Radiobiologiia 24, 697-700.

Richardson D.B., Wing S. (2007) Leukemia mortality among workers at the Savannah River Site, Am. J. Epidemiol. 166, 1015-1022.

Rossi H.H., Zaider M. (1990) Contribution of neutrons to the biological effects in Hiroshima, Health Phys. 58, 645-647.

Satow Y., Hori H., Lee J.Y. (1989) Teratogenic effect of fission neutron and tritium water on rat embryo, J. Uoeh 11(Suppl), 416-431.

Seyama T., Yamamoto O., Kinomura A., Yokoro K. (1991) Carcinogenic effects of tritiated water (HTO) in mice: in comparison to those of neutrons and gamma-rays, J. Radiat. Res. (Tokyo) 32(Suppl 2), 132-142.

Straume T. (1995) High-energy gamma rays in Hiroshima and Nagasaki: implications for risk and WR, Health Phys. 69, 954-956.

Straume T., Carsten A.L. (1993) Tritium radiobiology and relative biological effectiveness, Health Phys. 65, 657-672.

Streffer C., van Beuningen D., Elias S. (1978) Comparative effects of tritiated water and thymidine on the preimplanted mouse embryo in vitro, Curr. Top. Radiat. Res. $Q$ 12, 182-193.

Ueno A., Furuno-Fukushi I., Matsudaira H. (1989) Cell killing and mutation to 6-thioguanine resistance after exposure to tritiated amino acids and tritiated thymidine in cultured mammalian cells, In S. Okada (Ed.) Tritium Radiobiology and health Physics, Proceedings of the 3rd Japanese-US workshop, Nagoya University, Japan. IPPJ-REV-3, pp. 200-210.

Ujeno Y. (1983) Relative biological effectiveness (RBE) of tritium beta rays in relation to dose rate, Health Phys. 45, 789-791.

UNSCEAR (1977) Sources and effects of ionizing radiation. United Nations Scientific Committee on the Effects of Atomic Radiation, 1977 report to the general assembly, United Nations Publications, New-York.

UNSCEAR (1982) Sources and effects of ionizing radiations. United Nations Scientific Committee on the Effects of Atomic Radiation, 1982 report to the general assembly, United Nations Publications, New-York.

UNSCEAR (1993) Sources and effects of ionizing radiations. United Nations Scientific Committee on the Effects of Atomic Radiation, 1993 report to the general assembly, United Nations Publications, New-York.

Young R., Kerr G. (1995) Reassessment of the atomic bomb radiation dosimetry for Hiroshima and Nagasaki: Report of the joint US-Japan working group, Radiation Effects Research Foundation Hiroshima.

Zablotska L.B., Ashmore J.P., Howe G.R. (2004) Analysis of mortality among Canadian nuclear power industry workers after chronic low-dose exposure to ionizing radiation, Radiat. Res. 161, 633-641.

Zhou X.Y., Dong J.C., Zhou S.Y., Chen J.D., Guo F.R. (1989) Experimental study on relative biological effectiveness of tritium and risk estimates of genetic damage, Chin. Med. J. (Engl) 102, 872-878. 